\title{
Coercivity Enhancement in Exchange Biased Systems Driven by Interfacial Magnetic Frustration
}

\author{
C. Leighton, J. Nogués,* B. J. Jönsson-Åkerman, and Ivan K. Schuller \\ Physics Department 0319, University of California-San Diego, La Jolla, California 92093-0319
}

(Received 14 September 1999)

\begin{abstract}
We report the temperature and cooling field dependence of the coercivity of exchange biased $\mathrm{MnF}_{2} / \mathrm{Fe}$ bilayers. When the antiferromagnetic surface is in a state of maximum magnetic frustration and the net exchange bias is zero, we observe a strong enhancement of the coercivity, which is proportional to the exchange coupling between the layers. Hence, the coercivity can be tuned in a reproducible and repeatable fashion in the same sample. We propose that a frustrated interface provides local energy minima which effectively pin the propagating domain walls in the ferromagnet, leading to an enhanced coercivity.
\end{abstract}

PACS numbers: $75.70 . \mathrm{Cn}, 75.30 . \mathrm{Gw}$

The magnetic coercivity $H_{C}$ (i.e., the half-width of the magnetic hysteresis loop) is an important parameter used to characterize magnetic materials. Control over the coercivity is desirable to tune the behavior of magnetic devices. In spite of this, the issue of controlling the magnetic coercivity has received little attention from physicists as it is supposed to be an extrinsic quantity often determined by such parameters as defect density. As such it is difficult to control in a reproducible fashion by changing an external parameter. Exchange bias $H_{E}$ (the shift of the hysteresis loop along the field axis) has been extensively studied in antiferromagnetic (AF)/ferromagnetic (F) bilayers, although a quantitative understanding is still unavailable [1]. Despite this, some intriguing correlations exist between $H_{E}$ and $H_{C}$. Moreover, recent theoretical work [2,3] claims that the behavior of $H_{C}$ and the correlations between $H_{E}$ and $H_{C}$ provide important clues as to the microscopic origin of exchange anisotropy. However, experimental investigations of $H_{C}$, as well as $H_{E}$, in systems with well controlled and characterized microstructure are rare ([4-7] are examples).

Here we present measurements on an exchange biased system in which the coercivity can be tuned by the field applied $\left(H_{\mathrm{FC}}\right)$ when cooling through the AF Néel temperature $T_{N}$. In this fashion, the AF surface spin structure can be varied and its effect on the behavior of $H_{E}$ and $H_{C}$ observed. The crossover from negative to positive $H_{E}$, with increasing $H_{\mathrm{FC}}$, is accompanied by an additional increase in the coercivity. This increase is in addition to that which occurs on cooling below $T_{N}$ and increases with the exchange coupling between the AF and $\mathrm{F}$ layers. The dependence of the exchange bias on cooling field and temperature can be analyzed using a simple model in which the AF surface spin structure is modified by the applied $H_{\mathrm{FC}}$, in the presence of an antiferromagnetic coupling between the $\mathrm{F}$ and AF layers [7-9]. We show that this enhancement is brought about by magnetic frustration at the AF/F interface, a result that has important implications for the physics of exchange biased systems.
$\mathrm{ZnF}_{2}(25 \mathrm{~nm}) / \mathrm{MnF}_{2}(60 \mathrm{~nm}) / \mathrm{Fe}(12 \mathrm{~nm}) / \mathrm{Al}(3 \mathrm{~nm})$ layers were deposited by electron beam evaporation. The nonmagnetic $\mathrm{ZnF}_{2}$ layer serves as a buffer between the $\mathrm{MgO}(100)$ substrate and the $\mathrm{AF} \mathrm{MnF}_{2}$ layer $\left(T_{N}=67 \mathrm{~K}\right.$ [8]), while the $\mathrm{Al}$ capping layer prevents oxidation. $\mathrm{ZnF}_{2}$, $\mathrm{Fe}$, and $\mathrm{Al}$ were deposited at $200^{\circ} \mathrm{C}, 150{ }^{\circ} \mathrm{C}$, and $150{ }^{\circ} \mathrm{C}$, respectively, whereas $\mathrm{MnF}_{2}$ was deposited at several temperatures in the $275-375^{\circ} \mathrm{C}$ range. Varying the substrate temperature during deposition of the $\mathrm{MnF}_{2}$ layer affords control over the roughness $(\sigma)$ of the $\mathrm{MnF}_{2} / \mathrm{Fe}$ interface [8] which in turn changes $H_{E}$. The pressure during fluoride deposition is around $6 \times 10^{-7}$ Torr. X-ray diffraction, grazing incidence reflectivity, and reflection high energy electron diffraction were used for structural characterization, while magnetic measurements were made with a SQUID magnetometer between 4.2 and $100 \mathrm{~K}$ and in fields up to $70 \mathrm{kOe}$. Remnant fields were minimized by heating the superconducting magnet above its transition temperature after application of large fields. They were then measured and accounted for by measuring the hysteresis loops of single Fe films. It is noted that it has been suggested that hysteresis loops may provide a lower bound for $H_{E}$ [10]. However, the absence of training effects, the reproducibility of the data, and earlier reversible measurements [11] all indicate that hysteresis loops provide a good measure of $H_{E}$.

$\mathrm{ZnF}_{2}$ and $\mathrm{MnF}_{2}$ layers have a body centered tetragonal structure with a (110) orientation perpendicular to the substrate surface, while the $\mathrm{Fe}$ overlayers are polycrystalline. The (110) fluoride reflection peak widths give a grain size equivalent to the thickness of the film. The roughness $(\sigma)$ determined from refinement of the grazing incidence reflectivity [12] is identified as the rms thickness fluctuation at the fluoride-Fe interface over the relatively long length scale probed by grazing incidence reflectivity. This can be controlled from $0.6 \mathrm{~nm}$ up to $\gtrsim 4-5 \mathrm{~nm}$ without significantly varying the full width at half maximum of the $\mathrm{MnF}_{2}$ (110) reflection or the rocking curve peak width, thus proving that the crystalline quality is not affected. As 
shown previously, $\mathrm{MnF}_{2} / \mathrm{Fe}$ layers with "smooth" interfaces $(\sigma<1.5 \mathrm{~nm})$ show positive $H_{E}$ (i.e., in the same direction as $H_{\mathrm{FC}}$ ) for cooling fields $\gtrsim 10 \mathrm{kOe}$ while layers with "rough" interfaces show only negative $H_{E}$ (i.e., in the opposite direction to $H_{\mathrm{FC}}$ ) [8].

Figure 1 shows the temperature dependence of $H_{E}$ and $H_{C}$ [defined as the half loop width at magnetic moment $(m)=0$ ] for two $H_{\mathrm{FC}}$ values for a sample with a smooth $\mathrm{MnF}_{2} / \mathrm{Fe}$ interface $(\sigma=0.6 \mathrm{~nm})$. For low cooling field $\left(H_{\mathrm{FC}}=2 \mathrm{kOe}\right)$ typical behavior is observed: The negative $H_{E}$ switches on close to $T_{N}$ eventually saturating below $30 \mathrm{~K}$ [Fig. 1(a)], while $H_{C}$ increases monotonically with decreasing temperature below $T_{N}$ [Fig. 1(b)]. The temperature dependence of $H_{C}$ for $\mathrm{ZnF}_{2} / \mathrm{Fe} / \mathrm{Al}$, also in Fig. 1(b), shows a rather low $H_{C}(<15 \mathrm{Oe})$ with a weak temperature dependence. These data clearly indicate that the large coercivities at $T<T_{N}$ in the $\mathrm{MnF}_{2} / \mathrm{Fe}$ bilayers are due to exchange coupling across the AF/F interface. For $H_{\mathrm{FC}}=70 \mathrm{kOe}$ the temperature dependence of the positive $H_{E}$ [Fig. 1(c)] and $H_{C}$ [Fig. 1(d)] is similar to the $H_{\mathrm{FC}}=2 \mathrm{kOe}$ case. It is interesting that in both cases the $H_{C}$ enhancement below $T_{N}$ does not saturate at low temperatures, despite the fact that $H_{E}$ reaches a temperature independent value (presumably this reflects the saturation of the AF sublattice magnetization and anisotropy [13]). Such behavior in $H_{E}$ and $H_{C}$ has been observed in several systems (e.g., [4-6,14-16]) and appears to be a common phenomenon. It seems clear that this behavior warrants further study. We note that the theoretical work mentioned earlier [2] suggests that $H_{E}$ and $H_{C}$ are of fundamentally different origins $\left(H_{C}\right.$ is due to the uniaxial anisotropy resulting from spin-flop coupling while $H_{E}$ is created via a different mechanism,
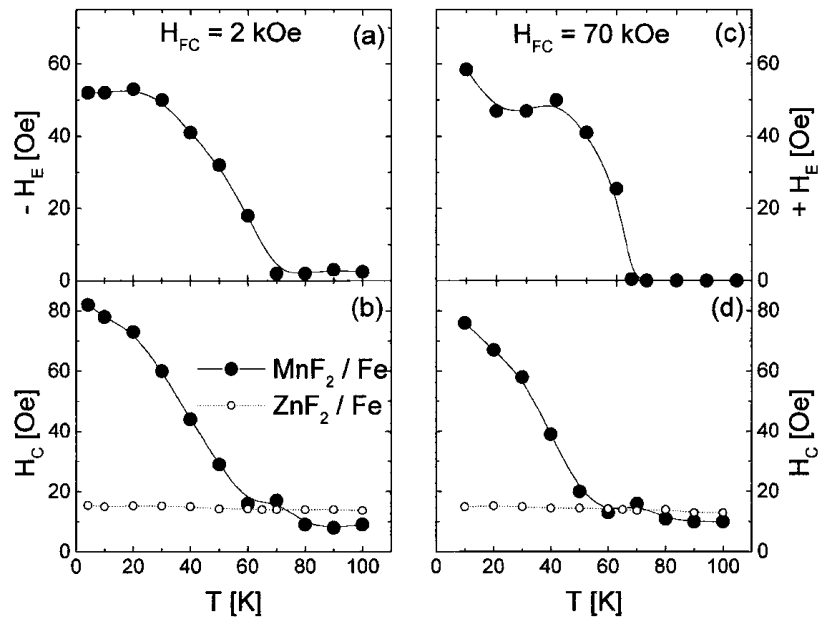

FIG. 1. $\quad H_{E}$ and $H_{C}$ as a function of $T$ for $H_{\mathrm{FC}}=2 \mathrm{kOe}$ and $H_{\mathrm{FC}}=70 \mathrm{kOe}$ [the sample has a smooth $\mathrm{MnF}_{2} / \mathrm{Fe}$ interface ( $\sigma=0.6 \mathrm{~nm})]$. (a) $H_{E}(T)$ for $H_{\mathrm{FC}}=2 \mathrm{kOe}$, (b) $H_{C}(T)$ for $H_{\mathrm{FC}}=2 \mathrm{kOe}$, (c) $H_{E}(T)$ for $H_{\mathrm{FC}}=70 \mathrm{kOe}$, and (d) $H_{C}(T)$ for $H_{\mathrm{FC}}=70 \mathrm{kOe}$. Note the sign reversal in $H_{E}$ from (a) to (c). Open symbols are for $\mathrm{ZnF}_{2} / \mathrm{Fe}$. The lines are guides to the eye. such as interfacial defects), suggesting that the temperature dependences should not necessarily be expected to be similar. However, we also note that identical mechanisms may give dissimilar temperature dependencies as coercivity is dependent upon thermally activated processes and is therefore expected to have a temperature dependence in the whole range of temperature measured here.

The cooling field dependence of $H_{E}$ and $H_{C}$ is shown in Fig. 2 for two representative samples: one with a smooth $\mathrm{MnF}_{2} / \mathrm{Fe}$ interface $(\sigma=0.6 \mathrm{~nm}$, shown in Fig. 1) and one with a rougher interface $(\sigma \approx 3 \mathrm{~nm})$. The smooth sample shows a crossover from negative to positive $H_{E}$ at $H_{\mathrm{FC}}=10 \mathrm{kOe}$, while the rough sample shows only negative exchange bias which is weakly dependent on $H_{\mathrm{FC}}$. This behavior was found to be due to a crossover from AF exchange coupling to $\mathrm{F}$ exchange coupling with increasing roughness [8]. The coercivity behavior is intriguing; the rough sample (which shows only negative $H_{E}$ ) exhibits a very weak dependence of $H_{C}$ on $H_{\mathrm{FC}}$ [Figs. 2(c) and 2(d)]. The smooth sample, on the other hand, shows a clear maximum in $H_{C}$ close to the $H_{\mathrm{FC}}$ for which $H_{E}=0$ [Figs. 2(a) and 2(b)]. The larger values of $H_{C}$ measured for the sample showing only negative $H_{E}$ are presumably due to the higher roughness of the Fe layer. Note that $H_{C}$ of $\mathrm{ZnF}_{2} / \mathrm{Fe}$ bilayers shows no measurable cooling field dependence.

Thus the cooling field provides an external agent by which the coercivity of the sample can be varied in a repeatable and reproducible way (i.e., the sample can be warmed above $T_{N}$, cooled to $10 \mathrm{~K}$ in the same field, and the same values of $H_{E}$ and $H_{C}$ recorded to within experimental uncertainty). In most other cases, changing $H_{C}$ requires changing the density or nature of the defects in
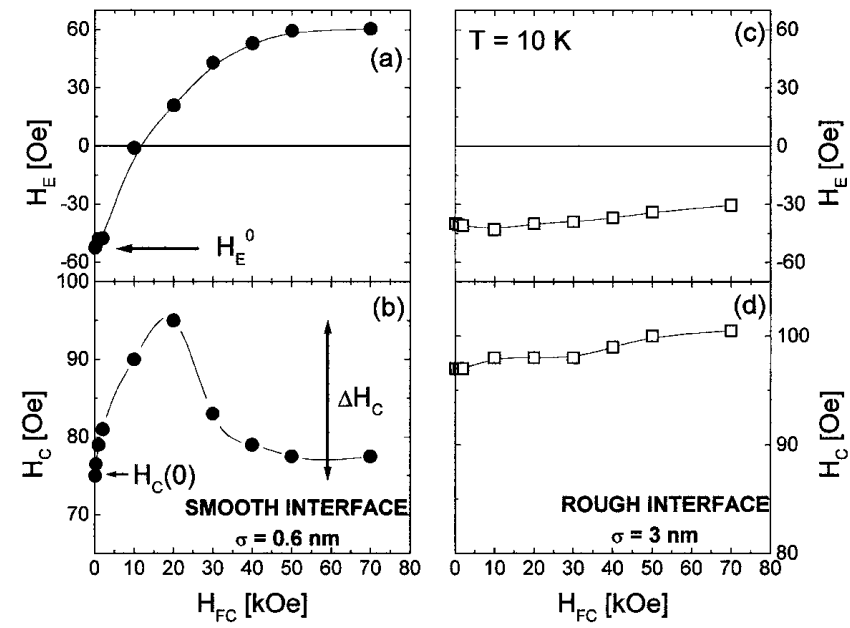

FIG. 2. $H_{E}$ and $H_{C}$ as a function of $H_{\mathrm{FC}}$ for a sample with a smooth $\mathrm{MnF}_{2} / \mathrm{Fe}$ interface $(\sigma=0.6 \mathrm{~nm}$; see Fig. 1) and a sample with a rougher interface $(\sigma \approx 3 \mathrm{~nm})$; (a) $H_{E}\left(H_{\mathrm{FC}}\right)$ for the smooth interface, (b) $H_{C}\left(H_{\mathrm{FC}}\right)$ for the smooth interface, (c) $H_{E}\left(H_{\mathrm{FC}}\right)$ for the rough interface, and (d) $H_{C}\left(H_{\mathrm{FC}}\right)$ for the rough interface. $T=10.0 \mathrm{~K}$. The lines are guides to the eye. 
the sample, or the crystallinity, with the consequent difficulties with interpretation.

We define the size of the "peak" in $H_{C}\left(H_{\mathrm{FC}}\right)$ [see Fig. 2(b)] as the increase of $H_{C}$ from $H_{\mathrm{FC}}=0$ to the maximum value of $H_{C}$, labeled $\Delta H_{C}$ - the "coercivity enhancement." The percentage value can then be defined as $\Delta H_{C} / H_{C}(0)$, where $H_{C}(0)$ is the coercivity at zero cooling field. Figure 3 shows this percentage coercivity enhancement plotted against the exchange bias measured at low $(2 \mathrm{kOe})$ cooling field $\left(H_{E}^{0}\right)$, for a total of seven samples. The variation in $H_{E}^{0}$ is achieved by varying the interfacial roughness via the substrate temperature during growth $[7,8]$. These seven samples exhibit AF coupling between the AF and $\mathrm{F}$ layers and the low cooling field exchange bias is indicative of the coupling strength across the interface [8]. Hence the data of Fig. 3 show that the coercivity enhancement is more pronounced for strong coupling between layers and approaches zero as the AF coupling strength falls to zero.

Since in exchange biased systems the $H_{C}$ enhancement is thought to originate from a finite $H_{E}$, the naive expectation is that $H_{C}$ should reach a minimum close to $H_{E}\left(H_{\mathrm{FC}}\right)=0$. However, Fig. 2 clearly shows that the maximum in $H_{C}$ occurs very close to the point at which $H_{E}\left(H_{\mathrm{FC}}\right)=0$. We first note that such coercivity maxima can generally be observed at magnetic phase transitions [17]. However, the AF surface or bulk spin flop can be ruled out as a possible mechanism as the fields involved are an order of magnitude larger than measured here [18]. The coercivity enhancement can be understood qualitatively and quantitatively as described below. Within the simple model for positive $H_{E}$ [7-9], the AF exchange coupling across the interface $\left(J_{\mathrm{F} / \mathrm{AF}}\right)$ is being frustrated by the coupling of the AF surface spins to $H_{\mathrm{FC}}$. At low cooling

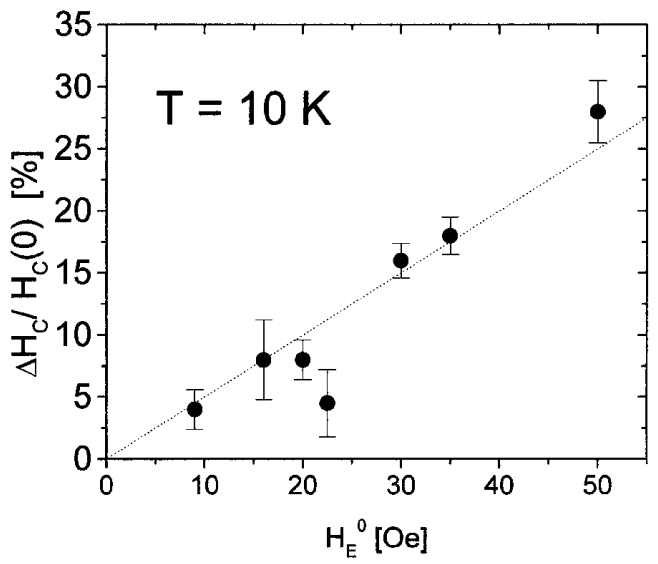

FIG. 3. $\Delta H_{C}$ (the percentage increase in coercivity over the $H_{\mathrm{FC}} \rightarrow 0$ value) as a function of $H_{E}^{0}$, the low cooling field exchange bias (measured at $H_{\mathrm{FC}}=2 \mathrm{kOe}$ ) for seven samples. The rms roughnesses as determined by low angle reflectivity are $0.60,0.72,0.80,0.92,1.00,1.13$, and $1.2 \mathrm{~nm}$. The dotted line is a least squares linear fit through the origin. $\Delta H_{C}$ and $H_{E}^{0}$ are labeled in Fig. 2. fields the AF coupling dominates and a low energy state is "frozen in" at $T_{N}$, but for high cooling fields the coupling of the AF surface spins to $H_{\mathrm{FC}}$ dominates and leads to an unstable state being frozen at $T_{N}$. This leads to the positive exchange bias. It is in the intermediate region where $H_{E}$ is close to zero that we observe the enhancement of the coercivity. Here some fraction of the spins are aligned with the cooling field and are therefore frustrating the AF exchange coupling, while others remain in the low potential energy, AF-coupled, state. It is at this point of maximum frustration that we observe the largest $H_{C}$. In other words, the AF surface splits into regions or "domains" [19], which are aligned either with $H_{\mathrm{FC}}$ or in the original AF-coupled configuration. Given that $H_{C}$ in these Fe films is dominated by domain wall pinning, an enhancement of $H_{C}$ can be understood in terms of increased pinning of the Fe domain walls at the edges of the AF domains. This explanation is also consistent with the data of Fig. 3 where it is seen that the coercivity enhancement becomes more pronounced with increasing exchange coupling. Essentially, the effectiveness of the $\mathrm{AF}$ domain walls as pinning sites for the propagating $\mathrm{F}$ domain wall increases as the exchange coupling between the layers is increased.

A simple modeling of this scenario can be realized by considering a domain wall in an $\mathrm{F}$ layer (in the $x-y$ plane) of thickness $t$, domain wall width $W$, and length $L$, propagating over a semi-infinite $\mathrm{AF}$ block split into $N$ square shaped domains aligned parallel or antiparallel to $H_{\mathrm{FC}}$. Balancing the energy due to an applied field $H$ with the energy change of the domain wall on propagating a distance $\delta y$ gives the following integral [20]:

$$
\begin{aligned}
2 M_{S} H L t=\frac{d \gamma}{d y_{0}}= & \int_{x_{0}-L / 2}^{x_{0}+L / 2} \gamma\left(x, y_{0}+W / 2\right) \\
& -\gamma\left(x, y_{0}-W / 2\right) d x,
\end{aligned}
$$

where $M_{S}$ is the saturation magnetization and $\gamma$ is the domain wall energy. $\quad \gamma$ can be written $\gamma(x, y)=$ $-M_{S} a_{0} \underline{\hat{m}}(x, y) \underline{h}(x, y)$, where $\underline{\hat{m}}(x, y)$ is a unit vector in the direction of the local magnetization, $\underline{h}(x, y)$ is the local field, and $a_{0}$ is the interfacial atomic separation. We then define $\underline{h}(x, y)$ as a series of delta functions at each domain edge which crudely models the real situation where the domain walls in the AF provide a local energy perturbation for the propagating $\mathrm{F}$ domain wall. Evaluating the integral we obtain $\Delta H_{C}=a_{0} h_{0} L / t d_{\mathrm{AF}}$, where $\Delta H_{C}$ is the enhancement of $H_{C}, d_{\mathrm{AF}}$ is the AF domain size, and the field $h_{0}$ is the delta function weight. To evaluate $\Delta H_{C}$ a suitable value for $h_{0}$ is required. As a first approximation, for an order of magnitude estimate we simply use the amplitude of the Malozemoff random field [21], $h_{0}=J_{\mathrm{AF} / \mathrm{F}} / M_{S} a_{0}^{3}$, giving

$$
\Delta H_{C}=J_{\mathrm{AF} / \mathrm{F}} L / d_{\mathrm{AF}} t M_{S} a_{0}^{2} .
$$

At low $H_{\mathrm{FC}}$ and high $H_{\mathrm{FC}}$ the $\mathrm{AF}$ is aligned antiparallel or parallel to $H_{\mathrm{FC}}$, there are no $\mathrm{AF}$ domains, $d_{\mathrm{AF}}$ approaches the sample size, and $\Delta H_{C}$ is minimized. At the 
point $H_{E}=0$ the number of $\mathrm{AF}$ domains reaches a maximum, hence $d_{\mathrm{AF}}$ reaches a minimum value and there is a consequent maximum enhancement in $H_{C}$. Taking literature values [22] for the parameters involved we obtain the reasonable value $d_{\mathrm{AF}} \sim 1000 \mathrm{~nm}$, required to reproduce our observed $\Delta H_{C}$ of $30 \%$. In addition to this, Eq. (2) implies that if the $\mathrm{AF}$ domain size $\left(d_{\mathrm{AF}}\right)$ is constant at the $H_{C}$ peak, the $H_{C}$ enhancement is directly proportional to $J_{\mathrm{AF} / \mathrm{F}}$. Given that $H_{E}$ is proportional to $J_{\mathrm{AF} / \mathrm{F}}$ [23] this dependence is exactly that observed in Fig. 3, where the $H_{C}$ enhancement increases linearly with the low field $H_{E}^{0}$.

In summary, we have measured the temperature and cooling field dependence of the coercivity of $\mathrm{MnF}_{2} / \mathrm{Fe}$ exchange biased bilayers. In this system the surface spin structure of the AF layer can be varied by the cooling field which systematically varies the exchange bias and coercivity. A strong coercivity enhancement is observed in the region where the AF surface spin structure exhibits maximal frustration. The coercivity enhancement is shown to be proportional to the exchange coupling between the layers. Simple modeling shows that the increased coercivity is due to enhanced pinning of the propagating domain wall in the F layer resulting from the interfacial magnetic frustration.

This work was supported by the U.S. Department of Energy. Discussions with E.D. Dahlberg, M. Kiwi, J. Mejía-López, R.D. Portugal, R. Ramírez, and M. R. Fitzsimmons are gratefully acknowledged. C.L. thanks S. Kim and M. Pardavi-Horvath for useful discussions and J. Santamaria for assistance with grazing incidence $\mathrm{x}$-ray simulations.

*Grup d'Electromagnetisme, Departament de Física, Universitat Autònoma de Barcelona, 08193 Bellaterra (Barcelona), Spain.

[1] For a recent review, see J. Nogués and I. K. Schuller, J. Magn. Magn. Mater. 192, 203 (1999).

[2] T. C. Schulthess and W. H. Butler, Phys. Rev. Lett. 81, 4516 (1998); J. Appl. Phys. 85, 5510 (1999).

[3] A. S. Carriço (private communication).

[4] R. Jungblut, R. Coehoorn, M. T. Johnson, Ch. Sauer, P. J. van der Zaag, A.R. Ball, Th. G.S.M. Rijks, J. aan de Stegge, and A. Reinders, J. Magn. Magn. Mater. 148, 300 (1996); R. Jungblut, R. Coehoorn, M. T. Johnson, J. aan de Stegge, and A. Reinders, J. Appl. Phys. 75, 6659 (1994).
[5] D. V. Dimitrov, S. Zhang, J. Q. Xiao, G. C. Hadjipanayis, and C. Prados, Phys. Rev. B 58, 12090 (1998).

[6] T. Ambrose, R. L. Sommer, and C. L. Chien, Phys. Rev. B 56, 83 (1997); X. W. Wu and C. L. Chien, Phys. Rev. Lett. 81, 2795 (1998); N. J. Gökemeijer and C. L. Chien, J. Appl. Phys. 85, 5516 (1999); T. Ambrose and C.L. Chien, J. Appl. Phys. 83, 6822 (1998).

[7] J. Nogués, D. Lederman, T. J. Moran, and I. K. Schuller, Phys. Rev. Lett. 76, 4624 (1996).

[8] C. Leighton, J. Nogués, H. Suhl, and I. K. Schuller, Phys. Rev. B 60, 12837 (1999).

[9] J. Nogués, C. Leighton, and I. K. Schuller, Phys. Rev. B 61, 1315 (2000).

[10] B.H. Miller and E. D. Dahlberg, Appl. Phys. Lett. 69, 3932 (1996); V. Ström, B. J. Jönsson, K. V. Rao, and E. D. Dahlberg, J. Appl. Phys. 81, 5003 (1998).

[11] E. D. Dahlberg, B. Miller, B. Hill, B. J. Jönsson, V. Ström, K. V. Rao, J. Nogués, and I. K. Schuller, J. Appl. Phys. 83, 6893 (1998).

[12] I. K. Schuller, Phys. Rev. Lett. 44, 1597 (1980); W. Sevenhans, M. Gijs, Y. Bruynseraede, H. Homma, and I. K. Schuller, Phys. Rev. B 34, 5955 (1986); E. E. Fullerton, I. K. Schuller, H. Vanderstraeten, and Y. Bruynseraede, Phys. Rev. B 45, 9292 (1992).

[13] U. Gäfvert, L. Lundgren, P. Nordblad, B. Westerstrandh, and O. Beckman, Solid State Commun. 23, 9 (1977).

[14] C. Schlenker, S. S. P. Parkin, J. C. Scott, and K. Howard, J. Magn. Magn. Mater. 54-57, 801 (1986).

[15] X. Lin, G. C. Hadjipanayis, and S. I. Shah, J. Appl. Phys. 75, 6676 (1994).

[16] H. Fujiwara, K. Nishioka, C. Hou, M. R. Parker, S. Gangopadhyay, and R. Metzger, J. Appl. Phys. 79, 6286 (1996).

[17] A. Berger, B. Feldman, H. Zillger, and M. Wuttig, J. Magn. Magn. Mater. 183, 35 (1998)

[18] J. Nogués, L. Morellon, C. Leighton, M. R. Ibarra, and I. K. Schuller, Phys. Rev. B (to be published).

[19] We use the term "domain" to refer to a region of the AF which is aligned parallel or antiparallel with $H_{\mathrm{FC}}$. We note that this is not the usual meaning of the term "AF domain."

[20] S. Zhang, D. V. Dimitrov, G. C. Hadjipanayis, J. W. Cai, and C.L. Chien, J. Magn. Magn. Mater. 198-199, 468 (1999).

[21] A. P. Malozemoff, J. Appl. Phys. 63, 3874 (1988).

[22] D. J. Craik, Magnetism (Wiley, New York, 1995), p. 404; A.S. Carrico, R.E. Camley, and R.L. Stamps, Phys. Rev. B 50, 13453 (1994).

[23] Most models of exchange bias agree that $H_{E}$ increases with $J_{\mathrm{AF} / \mathrm{F}}$ or $J_{\mathrm{AF}}$, although the exact dependence varies from model to model [1]. The simplest model predicts $H_{E} \propto J_{\mathrm{AF} / \mathrm{F}}[1]$. 